\title{
Three-Dimensional Primary Cell Culture: A Novel Preclinical Model for Pancreatic Neuroendocrine Tumors
}

\author{
Simon Leonhard April-Monn ${ }^{a, b}$ Tabea Wiedmer ${ }^{a, b} \quad$ Magdalena Skowronska $^{a}$ \\ Renaud Maire $^{a}$ Marco Schiavo Lena ${ }^{c}$ Mafalda Trippel ${ }^{a}$ \\ Annunziata Di Domenico $^{a, b}$ Francesca Muffatti $^{d}$ Valentina Andreasi $^{d}$ \\ Gabriele Capurso $^{d}$ Claudio Doglioni ${ }^{c, e}$ Corina Kim-Fuchs ${ }^{f}$ Beat Gloor ${ }^{f}$ \\ Maria Chiara Zatellig Stefano Partellid, e Massimo Falconid, e Aurel Perren ${ }^{a}$ \\ Ilaria Marinoni ${ }^{\mathrm{a}}$ \\ anstitute of Pathology, University of Bern, Bern, Switzerland; ${ }^{b}$ Graduate School for Cellular and Biomedical Sciences, \\ University of Bern, Bern, Switzerland; ' Unit of Pathology, San Raffaele Scientific Institute, Milan, Italy; ${ }^{\text {d Pancreatic }}$ \\ Surgery Unit, Pancreas Translational and Clinical Research Center, San Raffaele Scientific Institute, Milan, Italy; \\ e Università Vita e Salute, Milan, Italy; ${ }^{\mathrm{f}}$ Inselspital, University of Bern, Bern, Switzerland; ${ }^{9}$ Section of Endocrinology \\ and Internal Medicine, Department of Medical Sciences, University of Ferrara, Ferrara, Italy
}

\section{Keywords}

Pancreatic tumor - Neuroendocrine tumor - 3D culture .

Preclinical model · Primary cells · Drug screening · Islet-like tumoroids · Spheroids · Organoids

\begin{abstract}
Molecular mechanisms underlying the development and progression of pancreatic neuroendocrine tumors (PanNETs) are still insufficiently understood. Efficacy of currently approved PanNET therapies is limited. While novel treatment options are being developed, patient stratification permitting more personalized treatment selection in PanNET is yet not feasible since no predictive markers are established. The lack of representative in vitro and in vivo models as well as the rarity and heterogeneity of PanNET are prevailing reasons for this. In this study, we describe an in vitro 3-di-
\end{abstract}

mensional (3-D) human primary PanNET culture system as a novel preclinical model for more personalized therapy selection. We present a screening platform allowing multicenter sample collection and drug screening in 3-D cultures of human primary PanNET cells. We demonstrate that primary cells isolated from PanNET patients and cultured in vitro form islet-like tumoroids. Islet-like tumoroids retain a neuroendocrine phenotype and are viable for at least 2 weeks in culture with a high success rate (86\%). Viability can be monitored continuously allowing for a per-well normalization. In a proof-of-concept study, islet-like tumoroids were screened with three clinically approved therapies for PanNET: sunitinib, everolimus and temozolomide. Islet-like tumoroids

Simon Leonhard April-Monn and Tabea Wiedmer contributed equally to this work and shared first authorship. Aurel Perren and Ilaria Marinoni shared last authorship. karger@karger.com

(c) 2020 S. Karger AG, Basel

www.karger.com/nen

Karger ${ }^{\prime}=$
Ilaria Marinoni

Institute of Pathology, University of Bern

Murtenstrasse 31

CH-3008 Bern (Switzerland)

Ilaria.marinoni@pathology.unibe.ch 
display varying in vitro response profiles to distinct therapeutic regimes. Treatment response of islet-like tumoroids differs also between patient samples. We believe that the presented human PanNET screening platform is suitable for personalized drug testing in a larger patient cohort, and a broader application will help in identifying novel markers predicting treatment response and in refining PanNET therapy.

(c) 2020 S. Karger AG, Basel

\section{Introduction}

In contrast to many other malignancies, there are no molecular characteristics and biomarkers supporting treatment decisions in pancreatic neuroendocrine tumors (PanNETs). While molecular mechanisms underlying PanNET development and disease progression are continuously further deciphered $[1,2]$ and numbers of clinically approved therapies are steadily rising, the treatment options for PanNET are still primarily chosen based on clinician judgment.

The lack of appropriate models and the rarity of PanNET disease are two major factors that hinder further advances in PanNET translational research. Testing more effective therapies as well as performing predictive studies are lagging behind. Currently, only a limited number of human PanNET cell lines are available - with BON1, QGP1 and CM being the most commonly studied [3-6]. It is pertinent to note that while these cell lines were used to experimentally dissect molecular mechanisms of NETs, they do not represent well-differentiated slow-proliferating PanNETs. All of these cell lines are highly proliferative and were found to differ fundamentally in their mutational genetic background compared to PanNETs. In fact, studies have shown a strong resemblance to poorly differentiated pancreatic neuroendocrine carcinomas rather than PanNETs [7-11]. Moreover, other authors have even questioned the tissue of origin of the aforementioned cell lines, raising a significant debate as to the translational relevance of work performed using these cell lines $[11,12]$. Recently, Benten et al. [13] described NT-3 as a novel cell line that better reflects well-differentiated slow-proliferating PanNETs, which present the bulk of PanNETs. Nevertheless, the full molecular profile of NT-3 cells remains to be determined so that its similarity to the primary well-differentiated slow-proliferating subtype can be established. Moreover, neither available cell lines nor genetically engineered mouse models recapitulate the spectrum of different molecular subtypes found in human primary Pan-
NETs $[2,14]$. A stronger focus on developing more personalized in vitro models for studying these tumors is therefore demanded urgently. Cultivation and expansion of patient-derived neuroendocrine cells has been challenging owing to their intrinsically poor capacity for in vitro proliferation. However, due to advances in cell culture techniques, cell models of well-differentiated slow-proliferating PanNET derived from primary tissue have recently been used to study drug response and dissecting its underlying molecular mechanisms. For example, studies on isolated human primary PanNET cells cultured in vitro indicated that such a model might be utilized to determine patient response to treatment [1518]. However, the major limitations of all these studies are the short cultivation window of the cells, the nonphysiological 2-dimensional (2-D) format with limited cell-cell interactions, as well as their small scale in terms of investigated treatments and patient numbers.

In recent years, there have been tremendous advances in the development of 3-dimensional (3-D) tissue culture techniques, including scaffold-free setups in ultra-low attachment plates or scaffold-based encapsulation cultures to allow cell growth in 3 dimensions [19-23]. Culture of cells in 3 dimensions mimics a more physiological architecture of a tumor tissue, including cell-cell contact and allowing the development of spatial differences in the culture system with respect to proliferation, cell death and hypoxia within spheroids [21,24-26]. Additionally, cells kept in a 3-D format can be cultured and treated longer than in 2-D monolayers [21]. Cells cultured in 3 dimensions frequently display increased therapy resistance compared to cells cultured in 2 dimensions [2729], where 3-D culture most likely better reflects the in vivo situation [30, 31]. For this reason, lately highthroughput screenings of pharmacological compounds were preferentially performed in 3-D-cultured cells [3234].

With the presented study, we aimed for developing a platform to collect PanNET samples from multiple surgical centers, to isolate primary cells and to cultivate these cells in 3 dimensions retaining NET characteristics and finally to measure short- and long-term in vitro treatment response.

\section{Lead Contact and Material Availability}

Further information and request for resources and reagents should be directed to and will be fulfilled by the Lead Contact Dr. I. Marinoni (Ilaria.marinoni@pathology.unibe.ch). 


\section{Experimental Model and Subject Details}

\section{Primary Cell Culture}

Isolated primary PanNET cells were maintained in AdvDMEM + GF medium (DMEM-F12, 5\% FBS, Hepes $10 \mathrm{mM}, 1 \% \mathrm{~L}$-glutamine $(200 \mathrm{mM}), 1 \%$ penicillin $(100 \mathrm{U} /$ $\mathrm{mL}), 1 \%$ streptomycin $(0.1 \mathrm{mg} / \mathrm{mL}), 1 \%$ amphotericin B $(0.25 \mathrm{mg} / \mathrm{mL})$ (Merck, Switzerland), $20 \mathrm{ng} / \mathrm{mL}$ EGF, 10 ng/mL bFGF (Thermo Fisher Scientific, USA), $100 \mathrm{ng} / \mathrm{mL}$ PlGF, 769 ng/mL IGF-1 (Selleckchem, USA)) and in 24well Corning ${ }^{\circledR}$ Costar $^{\circledR}$ ultra-low attachment (ULA) plates (Corning, USA) $\left(500 \mu \mathrm{L} /\right.$ well, $3-5 \times 10^{5}$ cells/well) in a humidified cell incubator $\left(21 \% \mathrm{O}_{2}, 5 \% \mathrm{CO}_{2}, 37^{\circ} \mathrm{C}\right)$. For drug screen cells were resuspended in fresh AdvDMEM + GF medium supplemented with $123 \mu \mathrm{g} / \mathrm{mL}$ growth-factor-reduced Matrige ${ }^{\circledR}$ (Corning, USA) and plated in 96 -well ULA plates ( $50 \mu \mathrm{L} /$ well, $3-4 \times 10^{3}$ cells/ well).

To set up the PanNET screening platform including drug screening, we made use of primary material from a total of 16 PanNET patients depicted in online supplementary Table 1 (for all online suppl. material, see www. karger.com/doi/10.1159/000507669). Additionally, key resources used in this study are provided in Table 1.

\section{Patient Studies}

All patients agreed on the use of residual material and had signed an institutional informed consent. Patient characteristics are shown in online supplementary Table 1. The study was approved by the cantonal authorities (Kantonale Ethikkomission Bern, Ref.-Nr. KEK-BE 105/2015) and the Italian ethic commission (Comitato Etico, CE 252/2019).

\section{Methods}

\section{Patient Samples and Cryopreservation}

Fresh human PanNET tissue was obtained from patients diagnosed with PanNET undergoing surgery at the Inselspital Bern, Switzerland, or at the Pancreatic Surgery Unit, Pancreas Translational and Clinical Research Center, San Raffaele Scientific Institute, Milan, Italy. Tumor tissues of 16 PanNET patients were used for 19 isolations, 12 isolations for method establishment and 7 for a proofof-concept drug screening. Patient characteristics are summarized in online supplementary Table 1.

Upon surgical resection a pathologist processed one mirror block of the tumor tissue to $8-\mathrm{mm}^{3}$ blocks under sterile conditions avoiding necrotic regions. These blocks were suspended in recovery cell culture freezing medium (Thermo Fisher Scientific, USA), cryopreserved using an isopropyl alcohol freezing container (Nalgene, USA) and stored in liquid nitrogen. The other mirror block was embedded in a microcassette, and fixation was performed with a PAXgene Tissue System according to the manufacturer's instructions. In short, tissue was incubated in a PAXgene Tissue FIX Container (Qiagen, Germany) at room temperature overnight. Fixated tissue was transferred into a PAXgene Tissue FIX Container (Qiagen, Germany) at $4^{\circ} \mathrm{C}$ until paraffin embedding (1-2 days) or kept at $-20^{\circ} \mathrm{C}$ if embedding was not performed instantly.

\section{Primary Cell Isolation and Culture}

For primary cell isolation, tissue was thawed for 45-60 $\mathrm{s}$ in a $37^{\circ} \mathrm{C}$ water bath and cut into $1-\mathrm{mm}^{3}$ pieces and washed with medium (advanced DMEM-F12, Hepes 10 $\mathrm{mM}, 1 \%$ L-glutamine, $1 \%$ penicillin-streptomycin-amphotericin B) and Dulbecco's phosphate-buffered saline (DPBS; Thermo Fisher Scientific, USA). After aspiration of the medium, the tissue was incubated in $5 \mathrm{~mL}$ digestion medium (10 mg/mL collagenase IV (Worthington, USA), 0.25\% Trypsin-EDTA (Sigma-Aldrich, Switzerland), 10 $\mathrm{mg} / \mathrm{mL}$ DNase (Roche, Switzerland) in advanced DMEMF12, Hepes $10 \mathrm{mM}, 1 \% \mathrm{~L}$-glutamine, 1\% penicillin-streptomycin-amphotericin B) in a gentleMACS ${ }^{\mathrm{TM}}$ dissociator (Miltenyi Biotec, Switzerland) for $1 \mathrm{~h}$ at $37^{\circ} \mathrm{C}$ (programme TDK_1). After digestion, trypsin was deactivated with AdvDMEM (advanced DMEM-F12, 5\% FBS, Hepes $10 \mathrm{mM}, 1 \%$ L-glutamine, 1\% penicillin-streptomycin-amphotericin B), and cells were filtered through a 70$\mu \mathrm{m}$ smart strainer (Miltenyi Biotec, Switzerland) to remove debris of collagen. Red blood cells were lysed for 3 min with ACK lysis buffer (Thermo Fisher Scientific, USA) at room temperature. After $180 \mathrm{~g}$ centrifugation and aspiration of supernatant, the pellet was resuspended in AdvDMEM + GF medium (DMEM-F12, 5\% FBS, Hepes 10 mM, 1\% L-glutamine, 1\% penicillin-streptomycin-amphotericin B, $20 \mathrm{ng} / \mathrm{mL}$ EGF, $10 \mathrm{ng} / \mathrm{mL}$ bFGF (Thermo Fisher Scientific, USA), 100 ng/mL PlGF, 769 ng/ mL IGF-1 (Selleckchem, USA)). The cell suspension was plated in 24-well plates (cell + coated and tumor cells tested, Sarstedt, Germany) followed by a short spin for $30 \mathrm{~s}$, at $200 \mathrm{~g}$ and incubation for $1 \mathrm{~h}\left(21 \% \mathrm{O}_{2}, 5 \% \mathrm{CO}_{2}, 37^{\circ} \mathrm{C}\right)$ to partially segregate fibroblasts by attachment. The supernatant was collected. For single cell dissociation the cell suspension was transferred into a 5 - $\mathrm{mL}$ falcon tube and shortly spun down depending on cell/aggregate size. If large aggregates were present, cells were spun at 100-200 g; if smaller aggregates were present, cells were spun at 200 
Table 1. Key Resource Table

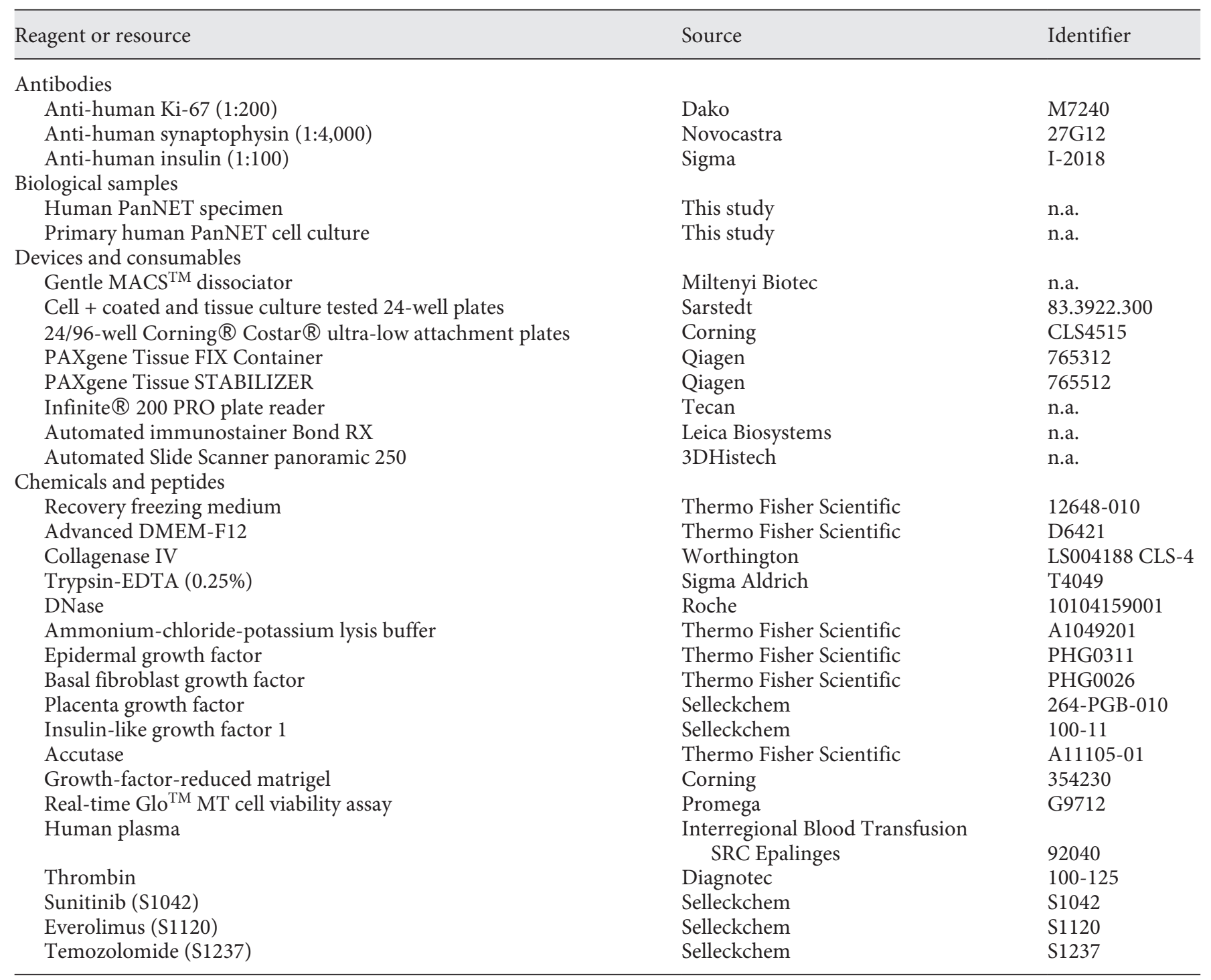

n.a., not available.

$300 \mathrm{~g}$. The cell pellet was washed with DPBS and incubated in Accutase (Thermo Fisher Scientific, USA) supplemented with DNase $(10 \mathrm{mg} / \mathrm{mL})$ (Thermo Fisher Scientific, USA). Cells were carefully dissociated by repeated $(10-15 \times)$ passage through a $1-\mathrm{mL}$ syringe and $26 \mathrm{G} 0.45 \times$ $13 \mathrm{~mm}$ Microlance $^{\mathrm{TM}}(\mathrm{BD}$, Switzerland) until aggregates were not visible anymore. The cell number was estimated using a hemocytometer, and cells were seeded in AdvDMEM + GF medium in 24-well ULA plates $\left(5 \times 10^{5}\right.$ cells $/ \mathrm{mL} /$ well). After 2 days of recovery phase, cellular aggregates were collected in $15-\mathrm{mL}$ falcon tubes and differ- entially centrifuged at $120 \mathrm{~g}$ for $5 \mathrm{~min}$ to separate cells and aggregates from debris/apoptotic cells. Supernatant was aspirated to remove semi- and nonviable cells. Cells were counted and resuspended in fresh AdvDMEM + GF medium supplemented with $123 \mu \mathrm{g} / \mathrm{mL}$ growth-factor-reduced Matrigel and plated in 96 -well ULA plates $(50 \mu \mathrm{L} /$ well, $3-4 \times 10^{3}$ cells/well). The setup consisted of 6-9 DMSO-positive control wells, 6 no-cell-negative control wells and technical triplicates for each drug concentration. 
Viability Measurement

RealTime-Glo ${ }^{\text {TM }}$ MT Cell Viability (RTG) assay (Promega, Switzerland) was used to repeatedly monitor cell viability in 3-D human primary PanNET culture. The RTG assay was performed according to the manufacturer's instructions, and luminescence was measured in an Infinite ${ }^{\circledR} 200$ PRO plate reader (Tecan, Switzerland). In brief, after 3 days of sphere formation, conditioned medium of each well was supplemented with additional 50 $\mu \mathrm{L}$ of fresh AdvDMEM + GF medium containing Matrigel and RTG assay reagents $(2 \times)$ to a final volume of 100 $\mu \mathrm{L}$. Growth factors and FBS were replenished from a concentrate $(0.77 \mu \mathrm{L}$ GFs $(130 \times)+5 \mu \mathrm{L}$ FBS $)$ every 3-4 days in each well using a multichannel pipette. A 6-h RTG baseline before adding drug compounds was recorded for every well at day 0 of the drug screen. For calculating the in vitro growth curve, relative luminescence unit (RLU) values were normalized to corresponding baselines. For calculating the in vitro drug response, RLU values were normalized first to corresponding baselines followed by normalization to the DMSO control wells of a particular day as described in more details in the paragraph "curve fitting and drug sensitivity data."

\section{Micro-Cell-Block from Islet-Like Tumoroids}

Islet-like tumoroids corresponding to $3-5 \times 10^{4}$ cells were collected in a $1.5-\mathrm{mL}$ Eppendorf tube (either directly on the day of isolation [D0] or from 6-9 wells of a 96-well ULA plate at the end of a drug screen [D15]). Tumoroids were washed with DPBS and pelleted at 350 $500 \mathrm{~g}$. The supernatant was aspirated, and the cells were resuspended in human plasma derived from whole blood (Interregional Blood Transfusion SRC, Epalinges, Switzerland) and Thrombin (Diagnotec, Switzerland) (ratio 5:1) followed by 3-min incubation at room temperature. The clot was fixed with 4\% PFA for 30-60 min protected from light. After a DPBS wash, the supernatant was aspirated and cells were incubated in a hematoxylin and DPBS solution (ratio 1:8) on a rocker shaker for 10-15 min at room temperature. The counterstained clot was transferred to a plastic microcassette for paraffin embedding. For immunohistochemistry the embedded material was cut into $2.5-\mu \mathrm{m}$-thick serial sections followed by deparaffinization, rehydration and antigen retrieval using an automated immunostainer (Bond RX, Leica Biosystems, Germany). Antigen retrieval was performed for Ki-67 (Dako M7240) with Tris for $30 \mathrm{~min}$ at $95^{\circ} \mathrm{C}$, insulin (Sigma I-2018) and synaptophysin (Novocastra 27G12) with Tris for $30 \mathrm{~min}$ at $100^{\circ} \mathrm{C}$. Antibodies were diluted as follows: Ki-67 1:200, insulin 1:4,000, syn-

Islet-Like Tumoroids: A Novel Preclinical PanNET Model aptophysin 1:100. Slides were counterstained with hematoxylin. Scans were acquired with an automated slide scanner Panoramic 250 (3DHistech, Hungary) at 20x magnification. Images were acquired using QuPath software [35].

\section{Drug Preparation}

Compounds (sunitinib (S1042), everolimus (S1120) and temozolomide (S1237)) were obtained from commercial vendors and stored as stock aliquots at $-80^{\circ} \mathrm{C}$. A 5-point, 625-fold concentration range was used for all compounds in order to have enough data points and a sufficient large drug concentration window to calculate reliable absolute $\mathrm{IC}_{50}$ values [36]. The starting dosage for each compound was selected based on $\mathrm{IC}_{50}$ screens in cancer cell lines publicly available online (see Cancerrxgene.org, PharmacoDB, Cancer Drug Resistance DB), from literature search and/or from in vitro data from pilot human primary cell cultures and/or from PanNET cell lines (QGP1, NT3, BON1).

\section{Curve Fitting and Drug Sensitivity Data}

Drug-response curve data consisted of 6-9 DMSOpositive controls, 6 no-cell-negative controls, and 5 drug-response points for a 625-fold concentration range. For $\mathrm{IC}_{50}$ calculation RLU values that were derived from an RTG assay from short-term treatment on day 3 and long-term treatment on day 7 of each well were weighted and normalized as the following: RLU values from each 6-h RTG baseline measurement $\left(R_{L} U_{x}\right.$ d0) were scaled with the overall minimal value of day 0 for each plate $\left(\mathrm{RLU}_{\min } \mathrm{d} 0\right)$ and transferred into a baseline weight $\left(R L U_{x}\right.$ weight $)$ for each well to minimize well-to-well variability:

$$
\mathrm{RLU}_{\mathrm{x}} \text { weight }=\frac{\mathrm{RLU}_{\mathrm{x}} \mathrm{d} 0}{\mathrm{RLU}_{\min } \mathrm{d} 0} .
$$

Each RLU value from day 3 was then accordingly weighted to its baseline weight:

$$
\mathrm{RLU}_{\mathrm{x}} \mathrm{d} 3 / 7 \text { weight }=\frac{\mathrm{RLU}_{\mathrm{x}} \mathrm{d} 3 / 7}{\mathrm{RLU}_{\mathrm{x}} \text { weight }} .
$$

The percentage response from weighted RLU was calculated by normalizing each value to no-cell-negative control (0\%) and DMSO-positive control (100\%) intervals. These data points were fitted in a 4-parametric linear-regression model $[34,37]$ with two constraints, top = $100 \%$ and bottom $=0 \%$, to estimate corresponding $\mathrm{IC}_{50}$. $\mathrm{IC}_{50}$ value differences of $>4$-fold were clustered in strongresponder and weak-responder groups. In case of an $\mathrm{IC}_{50}$ 
value $>2.5$-fold higher than the highest tested target-concentration samples were considered as non-responder (NR).

\section{Quantification and Statistical Analysis}

\section{QuPath Image Analysis}

Using QuPath software [35] digital-scanned hematoxylin-eosin and synaptophysin tissue sections were first preprocessed in the built-in visual stain editor using default settings for estimation of stain vectors to improve staining quality. Each tissue section was then superimposed with a 1,000- $\mu \mathrm{m}$ grid box. In each tissue section one representative tile out of the grid box was selected by a cytopathologist (M.T.) as a training set. Using a watershed segmentation method, positive and negative cells were automatically detected within each representative tile. Two pathologists (M.T., A.P.) then manually reconfirmed positive cell detection based on histomorphological features including cellular and nuclear shape, tumor cell nest formation, tumor columns, nuclear "salt and pepper" structure, nuclear and cytoplasmic staining intensity. A minimum of $\geq 1,000$ cells were selected for each training set and a total of 67 parameters (including perimeter, circularity, staining optical density, etc.) were included for training of the random-trees machine learning classifier. The auto-update tool within QuPath allowed real-time reconfirmation of training efficiency/accuracy. These cell detection parameters were applied on the whole tissue slides by creating a script which performed automated cell classification/annotation. Detection results were extracted from QuPath and imported and analyzed within $\mathrm{R}$.

Graph Pad Prism (Version 8.2.1) and R statistical environment were used for data analysis and visualization in the R version 3.6.2 (2019-12-12) platform: x86_64-w64mingw32/x64 (64-bit). Attached base packages: Grid; stats; graphics; grDevices; utils; datasets; methods; base. Other attached packages: 81) scales_1.1.0; (2) MASS_7.351.4; (3) reshape2_1.4.3; (4)ConsensusClusterPlus_1.50.0; (5) circlize_0.4.8; (6) ComplexHeatmap_2.2.0; (7) RColorBrewer_1.1-2; (8) Rmisc_1.5; (9) plyr_1.8.5; (10) lattice_0.20-38; (11) plotrix_3.7-7; (12) cowplot_1.0.0; (13) forcats_0.4.0; (14) stringr_1.4.0; (15) dplyr_0.8.3; (16) purrr_0.3.3; (17) tidyr_1.0.0; (18) tibble_2.1.3; (19) ggplot2_3.2.1; (20) tidyverse_1.3.0; (21) broom_0.5.3; (22) readr_1.3.1.

\section{Hierarchical Clustering Analysis of Drug Response} Profiles

Using the ConsensusClusterPlus pipeline [38], the number and membership of clusters was determined for drug response profiles based on patient-specific $\mathrm{IC}_{50}$ values of all three drug treatments. Distances were calculated using Pearson's correlation sorted by an agglomerative hierarchical clustering algorithm. The WardD2 algorithm was used for subsampling, and the final consensus matrix was determined by group average.

\section{Gene Expression Analysis}

Mean expression values of growth factor receptors were analyzed in publicly available data of 26 PanNET patients. RNAseq data were downloaded from the ICGC Data Portal (PAEN-AU project). QC, mapping/alignment and raw count quantification is described in Scarpa et al. [2]. From RSEM data output for our downstream analysis we chose the FPKM (fragments per kilobase of exon per million fragments mapped) normalization method to account for sequencing depth and gene length for all raw read counts. A list of all available growth factor receptor was acquired from the UniProt Knowledgebase [39]. Expression values of all targets were transformed into a 0 -to- 1 scale for each patient to allow interpatient comparability. Mean values for each target receptor were then calculated in all of the 26 PanNET patients:

Expression value $=$ mean $\left(\right.$ scaled $_{0.1}[$ FPKM normalized raw counts]).

$\chi^{2}$ Test and Monte-Carlo Simulation

A $\chi^{2}$ test of independence was conducted among all variables of interest. In order to meet requirements for $\chi^{2}$ test statistic and to account for relatively small expected cell frequencies our data set was resampled using a Monte Carlo simulation (replication $=1 \times 10^{5}$ ) allowing to calculate $p$ value estimates.

\section{Data and Code Availability}

The original RNAseq data set from human primary PanNET is publicly available at the ICGC Data Portal (PAEN-AU project). The complete expression data of growth factor receptors are available in the supplementary data sheet. The code supporting the current study has not been deposited in a public repository because the analysis code was generated from generic $\mathrm{R}$ packages, but the code is available from the corresponding author on request. 


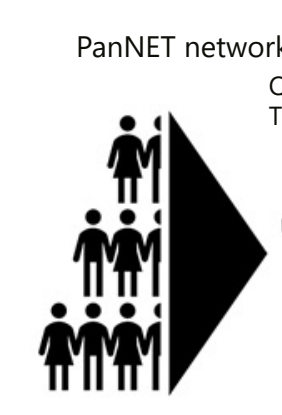

a
Mirror block

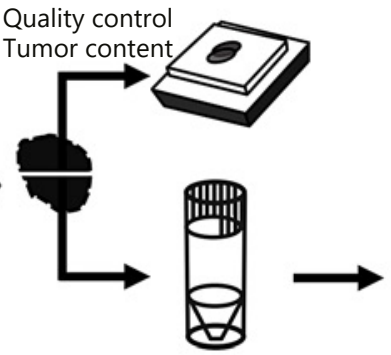

CRYO preservation
Drug-screening pipeline

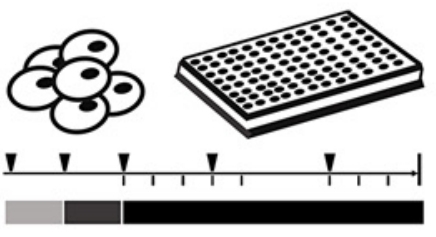

b

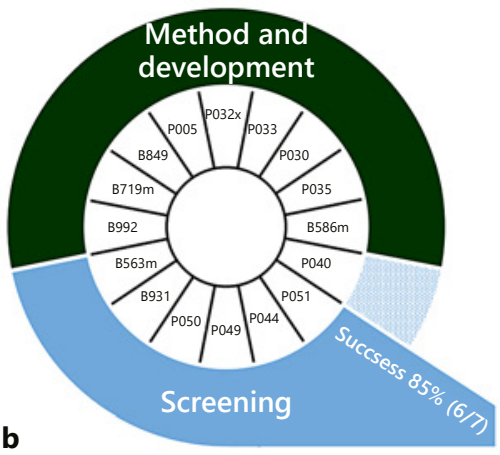

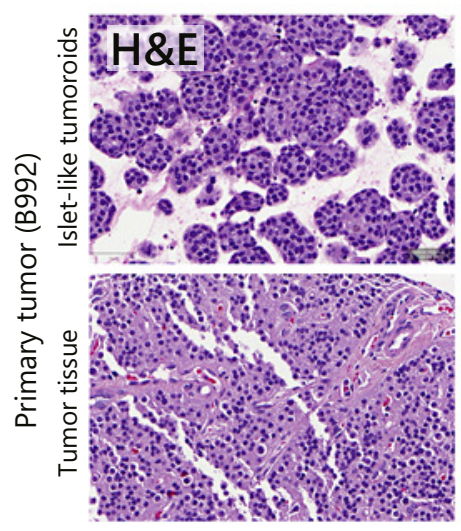
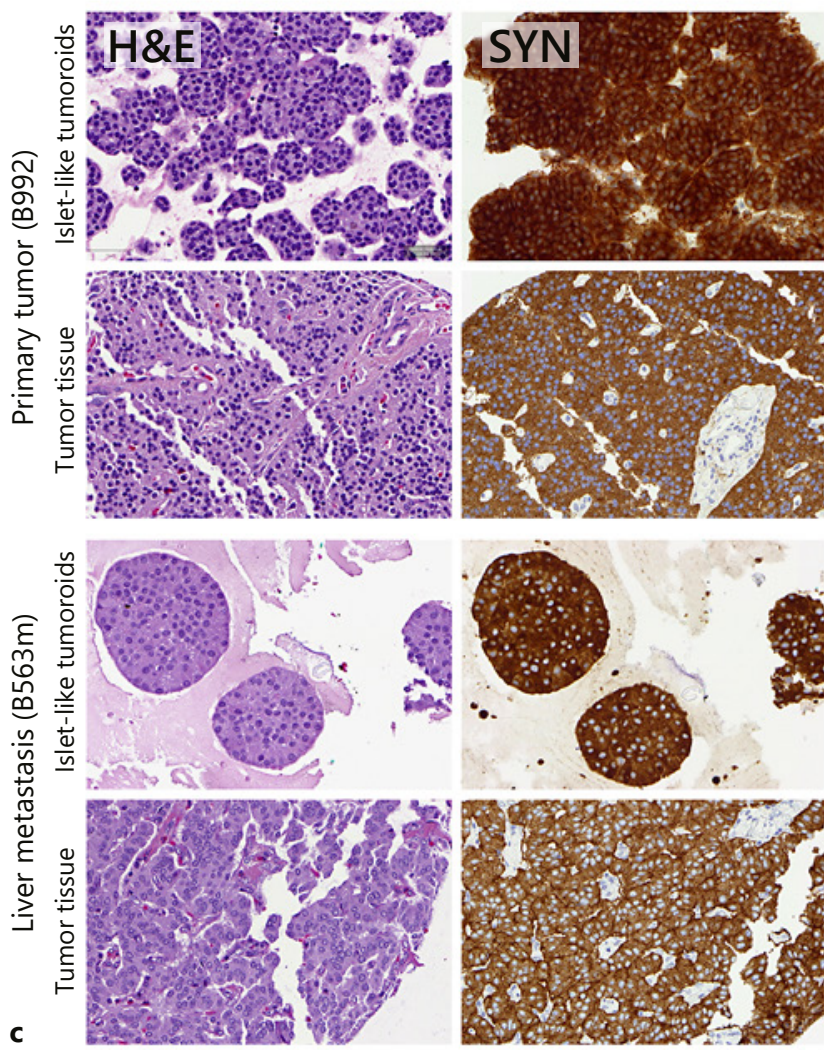
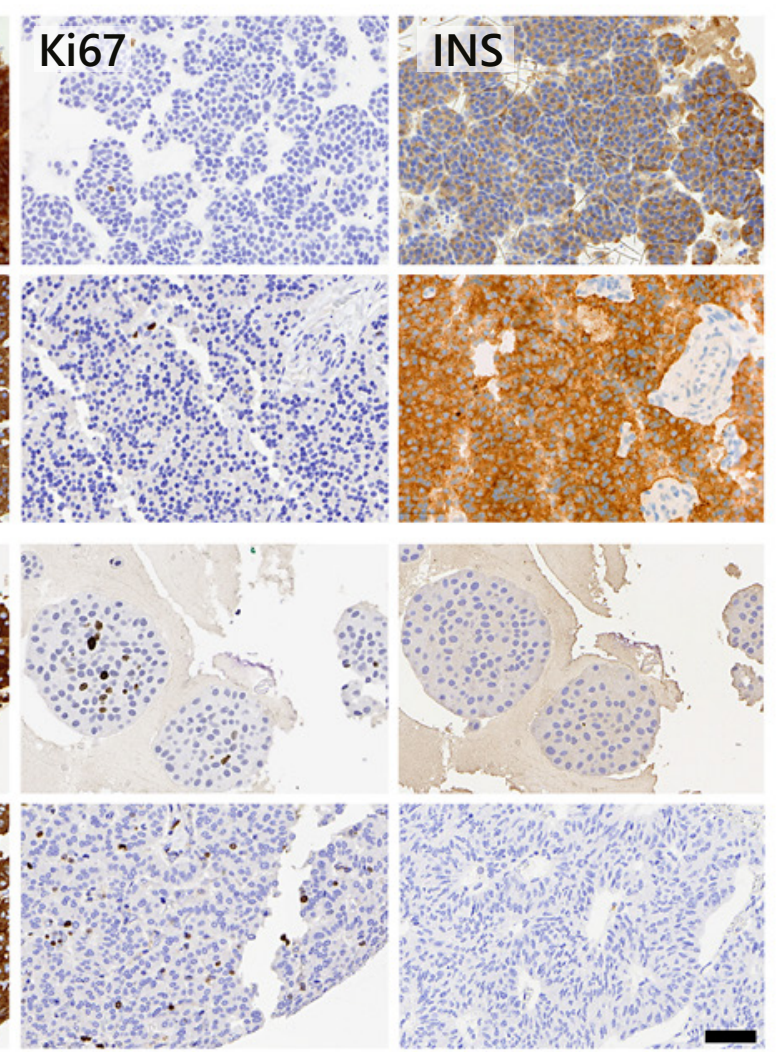

Fig. 1. Human primary PanNET cells form islet-like tumoroids and retain a neuroendocrine phenotype in vitro. a Schematic representation of human PanNET screening platform. b Venn diagram displaying usage of human PanNET patient material (outer circle) and individual patients (inner circle). The success rate for drug screening in PanNET patient material was 86\% (6/7). Detailed log of cell isolation is provided in the online supplementary data file 1. c Representative hematoxylin-eosin (HE) and immunohistochemistry staining of islet-like tumoroids from $\mathrm{B} 992$ and original primary tumor tissue B992 (top) and islet-like tumoroids from B563m and original metastatic tumor tissue from B563m (bottom). Cultured cells were formalin-fixed and embedded after 14 days in PanNET culture medium. Formalin-fixed primary PanNET tissue or cultured cells were stained for hematoxylin-eosin (HE), synaptophysin (SYN), insulin (INS) and Ki-67. Immunohistochemistry slides were counterstained with hematoxylin. Scale bar, $50 \mu \mathrm{m}$. 


\section{Results}

\section{Cryopreservation Allows a Multicenter Approach}

PanNET tumors are rare, therefore a collaborative network is crucial. Here we propose a novel platform for an effective multicenter approach which permits biobanking of cryopreserved PanNET tissues from multiple surgical centers by a single central institution which performs primary cell isolation and drug testing (Fig. 1a). After tumor resection, half of the specimen was formalin-free PAXgene-fixated and paraffin embedded. These so-called mirror blocks served as controls for sample quality and were used to preassess patient-specific PanNET characteristics and tumor cell content in hematoxylin-eosin stainings and synaptophysin immunohistochemistry - a NET biomarker routinely used in clinics for diagnosis of PanNET. The other half was immediately cryopreserved in recovery freezing medium and later shipped and processed for primary cell isolation and in vitro drug screening.

\section{Development of a 3-D Human Primary PanNET Cell Culture Model}

Within this study, we performed 19 isolations from samples of 16 PanNET patients. Patient characteristics are summarized in online supplementary Table 1 . In the first part of the study (12 patients), we set up the sampling, isolation and culture conditions. Two additional isolations were used for live-cell imaging to observe tumoroid formation. In the proof-of-concept part of the study (7 patients, including 3 patients who were also included in the method and development cohort), we tested the suitability of the setup for in vitro drug screening (Fig. 1b). During method development, we successfully isolated small aggregates and single cells from cryopreserved patient material in 73\% (8/11) (online supplementary data file 1). Age, sex and other clinical parameters such as WHO grade, TNM staging and Ki-67 indices did not reveal a significant association with the isolation success and/or cellular yield (online suppl. Fig. 1A).

In 3 patient samples, isolation did not yield aggregates or single cells, which we attribute to the collection of largely acellular fibrotic or necrotic tissue as revealed from hematoxylin-eosin staining of corresponding mirror blocks (online suppl. Fig. 1B). Therefore, careful macroscopic selection of viable cellular tumor regions is crucial. Upon implementation of SOPs for sampling at the surgical centers, we observed strong quality improvements. In 2 patient samples (P005, P051) yielding successful cell isolation, we detected overgrowth of fibro- blasts after 15 days (online suppl. Fig. 1C). This led us to implement a low FBS concentration in the culture medium and to include a partial fibroblast reduction step during cell isolation. Thus, the cell suspension was plated on coated plastic for $2 \mathrm{~h}$ followed by gently rinsing for the collection of low-adhesive nonstromal cells. Two tumor cell extracts (P032x, P033) were negative for synaptophysin immunohistochemistry (IHC) staining on conventional cytospin preparations and hence excluded for further culture. However, a post hoc analysis by a cytopathologist (M.T.) revealed that these cells were tumoral cells (online suppl. Fig. 1D). Consequently, to assess tumor cell content accurately, we implemented a formalinfixating paraffin-embedding technique termed MicroCell-Block. Micro-Cell-Blocks retain cellular- and tumoroid morphology and require only a low cell number. Micro-Cell-Blocks on the day of isolation (D0) served as an internal quality control to assess successful fibroblast removal and to guide decision for continuation of the drug screening pipeline. Micro-Cell-Blocks at the end of the experiment (D15) allowed to quantify tumor cell content from synaptophysin immunohistochemistry and to reconfirm target cell identity on hematoxylin-eosin staining.

To account for more physiological cell culture conditions a PanNET-specific culture medium was developed combining literature and human transcriptomic data from 26 low-grade PanNETs [2]. We selected growth factors that were frequently reported in the PanNET literature $[13,15,40]$ and for which - except of EGFR - all the target receptors (FGFR1, IGFR1/2, FLT1/VEGFR1, EGFR) were within the upper expression quintile $(<28 /$ 151) of all currently available growth factor receptors and related proteins [40] in human PanNET patients (online suppl. Fig. 1A). Additionally, PanNET culture medium was supplemented with a low percentage of extracellular matrix. Several findings showed improved in vitro culture from Matrigel complementation due to scaffolding support [41-45]. Low concentration Matrigel supplementation stabilized PanNET culture, without leading to a transient artificially increased cellular growth as seen with higher supplementation (data not shown).

\section{Human Primary PanNET Cells Form Islet-Like}

Tumoroids and Retain a Neuroendocrine Phenotype in vitro

After isolation and cell culture refinement we performed live-cell imaging in two human PanNET samples. We isolated single cells from cryopreserved primary PanNET tissue (B992) and PanNET liver metastasis (B563m). 


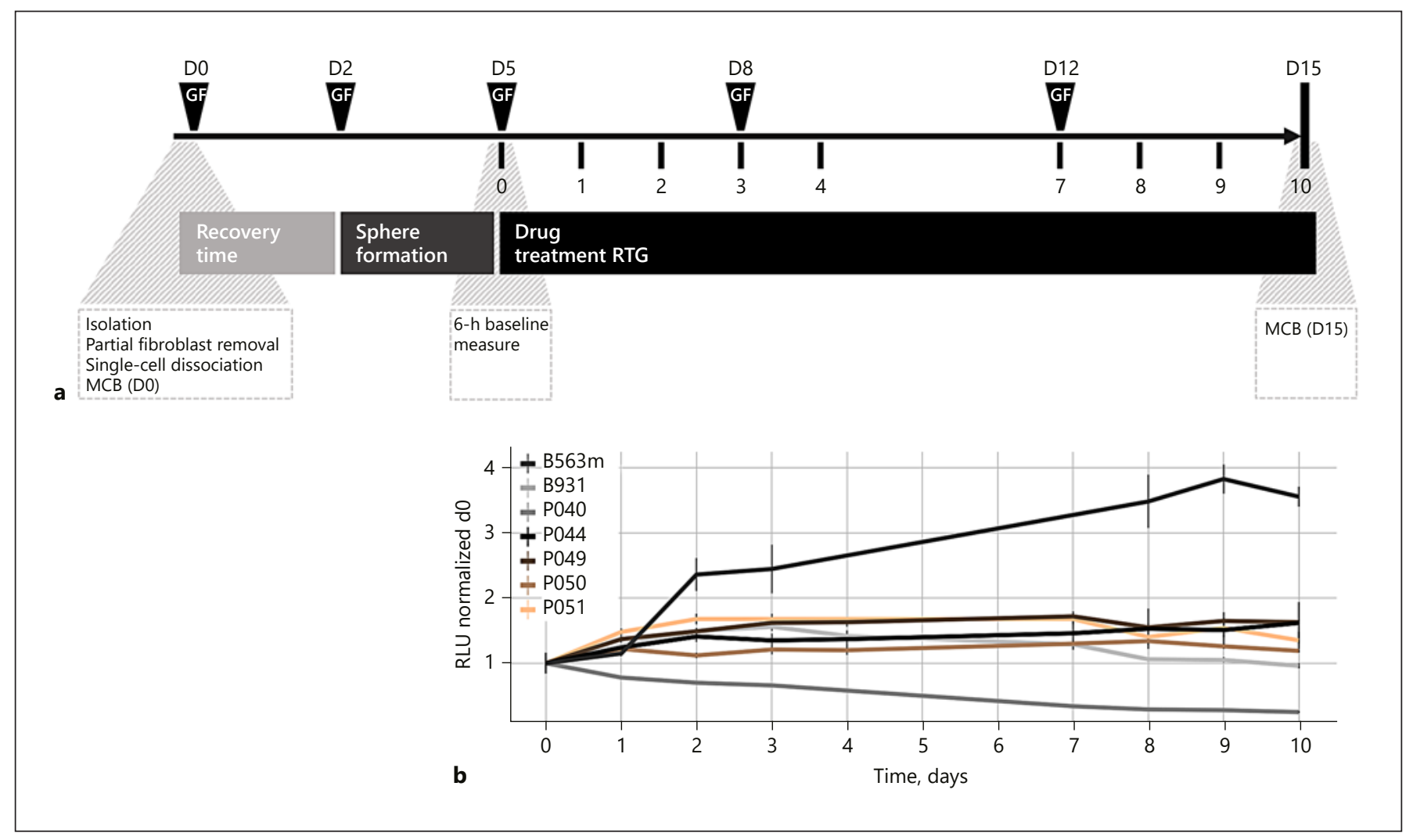

Fig. 2. 3-D human primary PanNET in vitro model for a personalized drug-screening platform. a Detailed schematic representation of in vitro 3-D drug-screening pipeline in human primary PanNET cells. GF, growth factor replenishment; thin ticks + digit, RTG viability assessment; MCB, Micro-Cell-Block. b In vitro growth curve of all screened primary PanNET samples using the metabolic surrogate assay RealTime-Glo (RTG) in 3-D human pri- mary PanNET culture. Cells were cultured in AdvDMEM + GF and a low percentage of matrigel in $0.16 \%$ DMSO for 10 days. Normalization was calculated based on per-well 6-h RTG baseline measurement. Data represent means $\pm \operatorname{SEM}(n=7,3$ technical replicates). RLU, relative luminescence unit.
Isolated cells from both patient specimens were viable. Live-cell imaging for 12 days revealed that isolated cells formed structures similar to extracted murine islets [46] which we hence termed islet-like tumoroids (online suppl. Fig. 2B). Islet-like tumoroids reached a more defined round structure after $72 \mathrm{~h}$ through aggregation and thereafter remained stable in volume (online suppl. Fig. 2C, suppl. video). Fourteen days after isolation, histomorphology of the islet-like tumoroids was examined and compared to corresponding mirror blocks. Islet-like tumoroids from primary and metastatic PanNET patient samples retained expression of synaptophysin, confirming that most of the cells consisted of tumor cells with preserved neuroendocrine phenotype (Fig. 1c). Furthermore, islet-like tumoroids from B992 expressed insulin as the original tumor tissue. The low percentage of Ki-67-positive tumor cells $(2 \%)$ in vitro matched with the prolifera- tion index described in the original tumor tissue (Ki-67 index $<2 \%$ ) (Fig. 1c, top, online suppl. Table 1). Similarly, the percentage of proliferating cells was retained in the metastatic PanNET sample (B563m), with a Ki-67 index of $12 \%$ in the original tumor tissues and $15 \%$ in cultured cells, respectively (Fig. 1c, bottom, online suppl. Table 1).

\section{Setting Up an in vitro Drug Screening Pipeline for} Islet-Like Tumoroids

After the successful pilot experiment, we sought to implement a pipeline for in vitro drug screening (Fig. 2a). Following a 2-day recovery phase after initial isolation, cells were replated from a 24 -well format into a 96-well format. By this time, the majority of semi- and nonviable cells from isolation had segregated from viable cells. As seen in previous live-cell imaging analysis (online suppl. Fig. 2B, C), during 72-h incubation in the 96-well plate, 


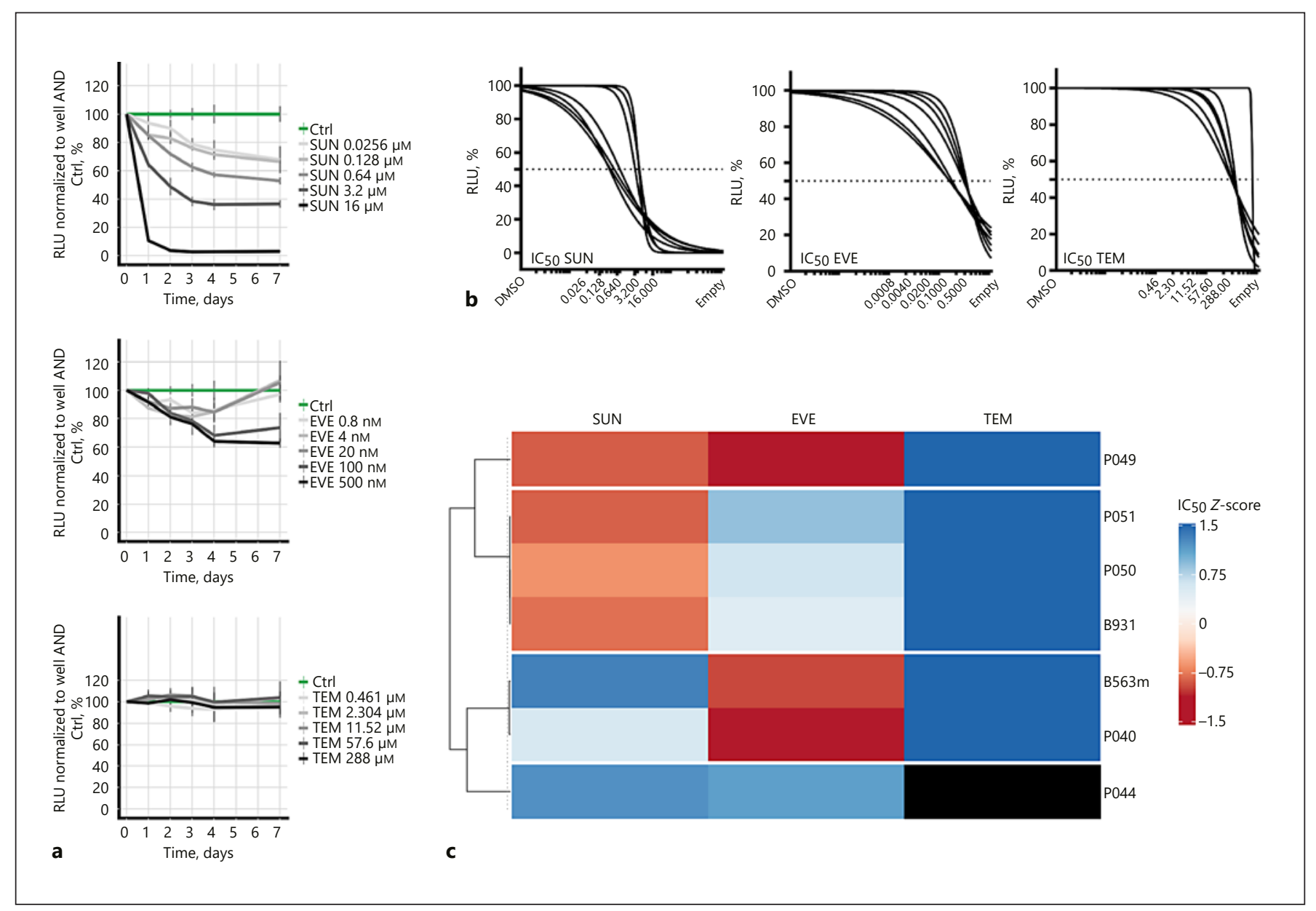

Fig. 3. Effect of standard of care pharmacological treatments on cell viability in 3-D human primary PanNET culture. a Representative in vitro viability curves using the metabolic surrogate assay RealTime-Glo (RTG) in 3-D human primary PanNET culture (P050) treated with $0.16 \%$ DMSO (control, Ctrl) or indicated treatment sunitinib (SUN), everolimus (EVE) and temozolomide (TEM) for 10 days. Normalization was calculated based on perwell 6-h RTG baseline measurement and corresponding DMSO control of the respective day. For all tested compounds a 5-point, 625 -fold concentration range was used based on vast literature research and in-house in vitro preliminary studies. Data represent means $\pm \operatorname{SEM}$ ( $n=1$ per patient, 3 technical replicates). RLU, rela-

cells formed islet-like tumoroids with only minor changes thereafter indicating a suitable time point for starting the drug treatment. Growth factors were replenished on days D2, D5, D8 and D12 after initial isolation. Due to low cell numbers available from PanNET specimens we selected RTG - a metabolic nonlytic assay - as a surrogate of cell viability. Pretreatment 6-h baseline measurements were recorded to normalize on an individual well basis and to correct for variability in cell number. Viability of tive luminescence unit. $\mathbf{b}$ In vitro dose response curves of screened PanNET patient displaying $\mathrm{IC}_{50}$ for SUN, EVE and TEM after short-term $(72 \mathrm{~h}$ ) treatment. Treatment responses (means \pm SEM) were fitted into a 4-parameter logistic regression model in GraphPad software to calculate absolute $\mathrm{IC}_{50}$. Data represent fitted curve means $(n=7)$. Dotted line, absolute $\mathrm{IC}_{50}$. $\mathbf{c}$ Heat map comparing absolute $\mathrm{IC}_{50} \mathrm{~s}$ for SUN, EVE and TEM in 3-D human primary PanNET culture after short-term $(72 \mathrm{~h})$ treatment. The heat map was derived using the WardD2 clustering method with displaying Pearson's clustering distance using ComplexHeatmap R-package [38]. The color code represents the scaled $\mathrm{IC}_{50}$ ( $Z$ score) for each drug. Each row represents an individual patient.

islet-like tumoroids in each well was repeatedly $(8 \times)$ monitored over a time course of 10 days before storing the cell material for further downstream analysis.

\section{PanNET Screening Pipeline in Control Conditions}

As a proof-of-concept experiment, we tested the PanNET drug-screening pipeline with 7 patient samples. Tumor cells were successfully isolated in all 7 PanNET specimens. Quantification of tumor cells in mirror blocks of 
original tumor tissue showed variable tumor content within tissues and among patients $(70 \pm 18 \%, n=7)$ (online suppl. Fig. 3A, B). Yet, assessment of hematoxylin-eosin stainings and synaptophysin immunohistochemistry on Micro-Cell-Blocks on the day of isolation (D0) by a cytopathologist (M.T.) reconfirmed successful selection of tumor cells after isolation and fibroblast depletion in all samples used for drug screenings ( $93 \pm 15 \%, n=7$ ) (online suppl. Fig. 3A, B). Moreover, successful culture of tumor cells was also reconfirmed in Micro-Cell-Blocks at the end of each experiment (D15) (95 $\pm 11 \%)$ (online suppl. Fig. 3A, B). In all patient samples, islet-like tumoroids were formed and remained viable in $85 \%$ (6/7) for 15 days in culture.

Following the metabolic activity of untreated islet-like tumoroids over the time course of 10 days, we observed an association between in vitro proliferation and Ki-67 index in original tumor tissue in the majority of samples: the metastatic patient sample (B563m) with a Ki-67 index of $15 \%$ in the original tumor tissue displayed the highest signal increase (3.8-fold), while in 4 patient samples with lower Ki-67 indices (P049, P050, P051, B931) the signal only increased between 1.1- and 1.7-fold (Fig. 2b). In patient sample P044 this association was weak exhibiting a 1.6-fold increase despite a Ki-67 index of $18 \%$ in the original tumor tissue. P040 was the only sample with a decreasing signal in the untreated condition; hence, longterm time points $(>72 \mathrm{~h})$ from this particular patient sample were not included in further analysis.

\section{In vitro Drug Response in Islet-Like Tumoroids Shows} Distinctive Sensitivity Profiles

To assess whether 3-D human primary PanNET culture could be exploited to predict patient drug response in vitro, we evaluated the effect of three clinically approved PanNET treatments on cell viability [47]. 3-D human primary PanNET cultures from 7 different patients were screened for response to sunitinib, everolimus and temozolomide. A 5-point, 625-fold drug concentration range ensured a sufficient exploratory drug screening window for accurate $\mathrm{IC}_{50}$ estimation based on mathematical modeling [36]. As starting points $\mathrm{IC}_{50}$ s from publicly available databases were interrogated for each drug, followed by pilot assessments of their antiproliferative effect in PanNET cell lines and murine primary cells (data not shown) as well as further literature research. Cells were treated for 10 days, and viability was repeatedly monitored at 8 time points during drug screening. Drug response profiles differed clearly among the three standard of care treatments. Dose-dependent effects of sunitinib and everolimus were observed in all tested patient sam-

Islet-Like Tumoroids: A Novel Preclinical PanNET Model ples (Fig. 3a, online suppl. Fig. 4A). Interestingly, comparing interpatient drug responses we detected varying treatment sensitivities among patients (online suppl. Fig. $4 \mathrm{~A}) . \mathrm{IC}_{50}$ values determined after short-term $(72 \mathrm{~h})$ treatment displayed two clearly distinct groups within sunitinib treatment and within everolimus treatment harboring $>4$-fold differences in respective $\mathrm{IC}_{50}$ (Fig. 3b). Also consensus clustering matrix and hierarchical cluster analysis $(\mathrm{k}=4)$ displayed robust response groups for short-term (72 h) treatments (Fig. 3c): a strong-responder group with samples sensitive to both treatments (P049), a group responding either primarily to everolimus (P049, P040, B563m) or to sunitinib (P050, B931, P051) - which was considered as mixed-responder group - and a weakresponder group including one sample insensitive to all treatments (P044). Importantly, in an integrative hierarchical cluster analysis, short-term treatment $\mathrm{IC}_{50} \mathrm{~s}-$ for the majority of patients - clustered closely together with long-term treatment $\mathrm{IC}_{50} \mathrm{~s}$ emphasizing robustness of the readouts (online suppl. Fig. 4B). Interestingly, in 1 case (B931) differences between short-term and long-term treatment were detected (online suppl. Fig. 4B).

\section{Discussion/Conclusion}

Current murine and human cell line models do neither accurately represent human well-differentiated slow-proliferating PanNETs, nor distinct human molecular subtypes, nor interpatient variability. In this study, we present a human PanNET screening platform allowing multicenter sample collection of cryopreserved patient specimens with an $86 \%$ success rate in primary cell isolation and cell culture. Isolated cells of well-differentiated slow-proliferating PanNET can be cultured in 3 dimensions and screened in vitro assessing response profiles to standard of care treatments for PanNETs. Since the cell number was the major limiting factor, we established protocols that are optimized for minimal amounts of resection specimens.

We present cryopreservation as a solution to make multicenter studies possible, thereby overcoming the issue of the rarity of PanNET samples. While difficult to implement in different centers, this generation of "living cell repositories" is promoted as innovative biobanking setting [48] and increasingly used in translational cancer research [49]. To account for more physiological conditions, growth factor supplementation for our PanNET culture medium was based on a combination of literature research $[13,15,40]$, transcriptomic analysis of growth factor receptors as well as pilot experiments testing different growth factor concen- 
trations and combinations. Final PanNET culture medium composition was selected according to best retention of viability during a 15-day period to minimize selection. This approach is clearly different from classical organoid approaches, where the culture medium selects for stem celllike cells, and where these cells are kept individually in a biomatrix in order to produce clonal organoids $[50,51]$. The aim of our presented "tumoroid" model is to in vitro treat a similar tumor cell composition as present in the patient. Following this approach, we can obtain a remarkable success rate of $86 \%$, but we acknowledge that classical organoid models have many other advantages such as the potential to intervene mechanistically [52-54]. With a retention of $\pm 70 \%$ of isolated cells in experiments before drug screening we believe that selection bias is minimal and that we are capable of treating the majority of cells representing the original tumor [55]. Compared to the limited number of studies using primary PanNET tissues in 2-D culture [15-18], we observe a reproducibly extended life span of the isolated cells up to 15 days. Longer experiments would also be possible at least in a subgroup of tumors; however, typically we did observe major responses already during the first 3 days of treatment.

We show that primary cells isolated from PanNET express original tumor characteristics and retain their neuroendocrine phenotype after 15 days. Interestingly, isolated cells form islet-like tumoroids in vitro. Similarly, nonneoplastic endocrine pancreatic cells are physiologically structured as islets. Kojima et al. reviewed the history of abundant findings which revealed that single cell suspension of endocrine pancreatic tissue from several species form islet-like structures and reconstitute their original architecture in vitro [56]. Currently, we do not know whether this reflects an endocrine specific phenotype or an even broader epithelial phenotype.

With the conditions presented, isolation and culture were successful in 6 out of 7 patients (86\%). Only 1 sample (P040) showed a loss of viability after 7 days and was excluded from further long-term treatment analysis. During 3-D culture, all G1 PanNET patient samples expectedly displayed only marginal growth, whereas the metastatic patient sample B563m (Ki-67 index of 15\%) showed the highest growth in vitro (3.8-fold in 10 days). One G2 sample (P044, Ki-67 index of 18\%) exhibited a somewhat lower growth of 1.6-fold in 10 days. It seems not surprising to infrequently observe a rather weak association of $\mathrm{Ki}-67$ index and in vitro proliferation. Indeed, a clear linear correlation of Ki-67 index to tumor growth rate has not been demonstrated clinically, to our knowledge. Biologically, proliferation represents only one aspect out of many: we neither have knowledge about a different fraction of cell death within our PanNET specimen nor do we know exact durations of cell cycles for the isolated cells from individual patient specimens.

In vitro treatment with clinically approved chemotherapeutics for advanced PanNET disease revealed overall distinctive response profiles and drug sensitivities based on $\mathrm{IC}_{50}$ s. Comparing short-term $(72 \mathrm{~h})$ versus long-term treatment ( 7 days and 15 days) showed identical results in the majority of samples, indicating that these different time windows are potentially of minor importance. However, in 1 tumor (B931) we observed differences between short-term and long-term treatment. While it might not be important to use long-term treatment for detecting primary response in sunitinib and everolimus treatment, prolonged treatment could be of potential importance for other chemotherapeutics. In our series, we do not see a clear response to temozolomide in all of the 7 PanNETs examined. A possible explanation for this is the mechanism of action of temozolomide, which is strongly linked to cellular proliferation. Cytotoxicity of temozolomide is mediated by $\mathrm{O}_{6}$-methylguanine adducts, which can mispair with thymine during DNA replication. The resulting futile cycles of DNA processing induce cytotoxic doublestrand DNA breaks that trigger apoptosis $[57,58]$. Due to the low proliferation rate of our samples a time window of 10 days may be still too short for a detection of measurable effects. In line with that, the metastatic patient sample (B563m) that proliferated in vitro shows at least a faint response to temozolomide in our screen - even if the $\mathrm{IC}_{50}$ estimation is still far from our tested drug concentration window (online suppl. Fig. 4A) and even if the sample has been scored as weak responder. We can exclude nonpotency of the chemotherapeutic compound itself since our implemented 625-fold concentration window (0.46$288.00 \mu \mathrm{M})$ covers a sufficiently large drug window tested in PanNET and glioblastoma cell lines (data not shown) to eliminate this as a potential bias.

In other tumor entities it has been shown that ex vivo drug response correlates with patients' response in primary cell culture approaches similar to our setup (e.g. esophageal adenocarcinoma, breast cancer, and head and neck squamous cell carcinoma) [59-61] and in patient-derived xenograft models $[62,63]$. Further studies are needed to evaluate whether the observed in vitro sensitivity will correlate with clinical response in PanNET patients as well. To answer this question, first a prolonged clinical follow-up is crucial. Secondly, a larger patient cohort will be needed to perform correlation analysis and to have enough statistical power. Full clinical follow-up data of all enrolled patients 
are therefore collected. Chemotherapy-specific in vitro treatment duration and concentration range with the highest predictive value will be defined by comparison of clinical data with the in vitro drug response. Due to the nonlytic approach, the islet-like tumoroids are collected after the experiment and are available for next-generation-sequencing end point analysis, as is the original tumor material from patients. The presented PanNET screening platform might therefore serve as a basis for developing personalized treatment of PanNET patients, performing molecular fingerprinting on the original tissue to be able to potentially detect predictive markers.

We are well aware that the present protocol still bears limitations. It depends on surgical resection specimens of PanNET metastases and high-stage tumors; however, most of these patients are diagnosed via biopsies of liver metastases. With further experience the protocol has the potential of being adapted to biopsy specimens as well yet - some biological role must be proven first to ethically justify additional biopsies. The composition of growth factors could be further refined, and the culture system does not factor in stromal and inflammatory features potentially contributing also to tumor response.

In conclusion, we present a 3-D human primary PanNET screening platform as a new preclinical model, which reflects the characteristics of an individual tumor and has the capability to detect differential treatment response. Therefore, this model has the potential to pave the way towards more personalized medicine for PanNET patients in the future, including better patient stratification and identification of novel and experimental treatments.

\section{Acknowledgment}

We thank the Tissue Bank Bern (Bern, Switzerland) and the Translational Research Unit (Institute of Pathology, Bern, Switzerland) for their technical, material and administrative support and the Cytopathology (Institute of Pathology, Bern, Switzerland) for technical support performing formalin-fixation and paraffin embedding in tissue and cell culture material. We also thank Dr. Joel Zindel, Mr. Philipp Zens and Mr. Hassan Sadozai for their constructive feedback on the manuscript drafts.

\section{Statement of Ethics}

Subjects (or their parents or guardians) have given their written informed consent, and the study protocol was approved by the cantonal authorities (Kantonale Ethikkomission Bern, Ref.-Nr. KEK-BE 105/2015) and the Italian ethic commission (Comitato Etico, CE 252/2019).

\section{Disclosure Statement}

All the authors declare no competing financial interest. There is no conflict of interest that could be perceived as prejudicing the impartiality of the research reported.

\section{Funding Sources}

The study was supported by Wilhelm Sander to Ilaria Marinoni and a Swiss Cancer League Grant (KLS 3360-02-2014) to Aurel Perren. Valentina Andreasi's PhD scholarship and Francesca Muffatti's research fellowship were supported by Gioja Bianca Costanza legacy donation.

\section{Author Contributions}

A.P., I.M., T.W. and S.L.A.M. designed the study. T.W. and S.L.A.M. developed the tumoroid methodology. T.W., M.S. and S.L.A.M. performed experiments and data acquisition. S.L.A.M. analyzed and visualized data. M.T. analyzed, scored and quantified immunohistochemistry. M.F., C.D., M.S.L., F.M., S.P., C.K.F. and B.G. provided patient biopsies and patient clinical information. R.M., M.S.L., A.D., V.A., M.C.Z., C.K.F., B.G. and M.S. provided administrative, technical or material support. A.P., I.M., T.W. and S.L.A.M. wrote the paper.

\section{References}

1 Di Domenico A, Wiedmer T, Marinoni I, Perren A. Genetic and epigenetic drivers of neuroendocrine tumours (NET). Endocr Relat Cancer. 2017 Sep;24(9):R315-34.

2 Scarpa A, Chang DK, Nones K, Corbo V, Patch AM, Bailey P, et al; Australian Pancreatic Cancer Genome Initiative. Whole-genome landscape of pancreatic neuroendocrine tumours. Nature. 2017 Mar;543(7643): 65-71.

3 Baroni MG, Cavallo MG, Mark M, Monetini L, Stoehrer B, Pozzilli P. Beta-cell gene expression and functional characterisation of the human insulinoma cell line CM. J Endocrinol. 1999 Apr;161(1):59-68.

4 Evers BM, Ishizuka J, Townsend CM Jr Thompson JC. The human carcinoid cell line, BON. A model system for the study of carcinoid tumors. Ann NY Acad Sci. 1994 Sep; 733(1):393-406.

5 Iguchi H, Hayashi I, Kono A. A somatostatinsecreting cell line established from a human pancreatic islet cell carcinoma (somatostatinoma): release experiment and immunohistochemical study. Cancer Res. 1990 Jun; 50(12):3691-3
6 Kaku M, Nishiyama T, Yagawa K, Abe M. Establishment of a carcinoembryonic antigenproducing cell line from human pancreatic carcinoma. Gan. 1980 Oct;71(5):596-601.

7 Doihara H, Nozawa K, Kojima R, KawabataShoda E, Yokoyama T, Ito H. QGP-1 cells release 5-HT via TRPA1 activation; a model of human enterochromaffin cells. Mol Cell Biochem. 2009 Nov;331(1-2):239-45.

8 Gragnoli C. The CM cell line derived from liver metastasis of malignant human insulinoma is not a valid beta cell model for in vitro studies. J Cell Physiol. 2008 Aug;216(2):569-70. 
9 Jonnakuty C, Gragnoli C. Karyotype of the human insulinoma CM cell line-beta cell model in vitro? J Cell Physiol. 2007 Dec; 213(3):661-2.

10 Lopez JR, Claessen SM, Macville MV, Albrechts JC, Skogseid B, Speel EJ. Spectral karyotypic and comparative genomic analysis of the endocrine pancreatic tumor cell line BON-1. Neuroendocrinology. 2010;91(2): $131-41$.

11 Siddique ZL, Drozdov I, Floch J, Gustafsson BI, Stunes K, Pfragner R, et al. KRJ-I and BON cell lines: defining an appropriate enterochromaffin cell neuroendocrine tumor model Neuroendocrinology. 2009;89(4):458-70.

12 Boora GK, Kanwar R, Kulkarni AA, Pleticha J, Ames M, Schroth G, et al. Exome-level comparison of primary well-differentiated neuroendocrine tumors and their cell lines. Cancer Genet. 2015 Jul-Aug;208(7-8):374-81.

13 Benten D, Behrang Y, Unrau L, Weissmann V, Wolters-Eisfeld G, Burdak-Rothkamm S, et al. Establishment of the First Well-differentiated Human Pancreatic Neuroendocrine Tumor Model. Mol Cancer Res. 2018 Mar; 16(3):496-507.

14 Sadanandam A, Wullschleger S, Lyssiotis CA, Grötzinger C, Barbi S, Bersani S, et al. A CrossSpecies Analysis in Pancreatic Neuroendocrine Tumors Reveals Molecular Subtypes with Distinctive Clinical, Metastatic, Developmental, and Metabolic Characteristics. Cancer Discov. 2015 Dec;5(12):1296-313.

15 Falletta S, Partelli S, Rubini C, Nann D, Doria A, Marinoni I, et al. mTOR inhibitors response and mTOR pathway in pancreatic neuroendocrine tumors. Endocr Relat Cancer. 2016 Nov;23(11):883-91.

16 Mohamed A, Romano D, Saveanu A, Roche C, Albertelli M, Barbieri F, et al. Anti-proliferative and anti-secretory effects of everolimus on human pancreatic neuroendocrine tumors primary cultures: is there any benefit from combination with somatostatin analogs? Oncotarget. 2017 Jun;8(25): 41044-63.

17 Mohamed A, Blanchard MP, Albertelli M, Barbieri F, Brue T, Niccoli P, et al. Pasireotide and octreotide antiproliferative effects and sst 2 trafficking in human pancreatic neuroendocrine tumor cultures. Endocr Relat Cancer. 2014 Oct;21(5):691-704.

18 Wiedmer T, Blank A, Pantasis S, Normand L, Bill R, Krebs P, et al. Autophagy Inhibition Improves Sunitinib Efficacy in Pancreatic Neuroendocrine Tumors via a Lysosome-dependent Mechanism. Mol Cancer Ther. 2017 Nov;16(11):2502-15.

19 Aboulkheyr Es H, Montazeri L, Aref AR, Vosough M, Baharvand H. Personalized Cancer Medicine: An Organoid Approach. Trends Biotechnol. 2018 Apr;36(4):358-71.

20 Broguiere N, Isenmann L, Hirt C, Ringel T, Placzek S, Cavalli E, et al. Growth of Epithelial Organoids in a Defined Hydrogel. Adv Mater. 2018 Oct;30(43):e1801621.
21 Nath S, Devi GR. Three-dimensional culture systems in cancer research: focus on tumor spheroid model. Pharmacol Ther. 2016 Jul; 163:94-108.

22 Tuveson D, Clevers H. Cancer modeling meets human organoid technology. Science. 2019 Jun;364(6444):952-5.

$23 \mathrm{Xu} \mathrm{H}$, Lyu X, Yi M, Zhao W, Song Y, Wu K. Organoid technology and applications in cancer research. J Hematol Oncol. 2018 Sep; 11(1):116

24 Huang L, Holtzinger A, Jagan I, BeGora M, Lohse I, Ngai N, et al. Ductal pancreatic cancer modeling and drug screening using human pluripotent stem cell- and patient-derived tumor organoids. Nat Med. 2015 Nov; 21(11):1364-71.

25 Pampaloni F, Reynaud EG, Stelzer EH. The third dimension bridges the gap between cell culture and live tissue. Nat Rev Mol Cell Biol. 2007 Oct;8(10):839-45.

26 Bresciani G, Hofland LJ, Dogan F, Giamas G, Gagliano T, Zatelli MC. Evaluation of spheroid 3D culture methods to study a pancreatic neuroendocrine neoplasm cell line. Front Endocrinol (Lausanne). 2019 Oct;10:682.

27 Ekert JE, Johnson K, Strake B, Pardinas J, Jarantow S, Perkinson R, et al. Three-dimensional lung tumor microenvironment modulates therapeutic compound responsiveness in vitro-implication for drug development. PLoS One. 2014 Mar;9(3):e92248.

28 Luca AC, Mersch S, Deenen R, Schmidt S, Messner I, Schäfer KL, et al. Impact of the 3D microenvironment on phenotype, gene expression, and EGFR inhibition of colorectal cancer cell lines. PLoS One. 2013;8(3):e59689.

29 Riedl A, Schlederer M, Pudelko K, Stadler M, Walter S, Unterleuthner D, et al. Comparison of cancer cells in $2 \mathrm{D}$ vs $3 \mathrm{D}$ culture reveals differences in AKT-mTOR-S6K signaling and drug responses. J Cell Sci. 2017 Jan;130(1):203-18.

30 McMillin DW, Negri JM, Mitsiades CS. The role of tumour-stromal interactions in modifying drug response: challenges and opportunities. Nat Rev Drug Discov. 2013 Mar;12(3):217-28.

31 Weiswald LB, Bellet D, Dangles-Marie V. Spherical cancer models in tumor biology. Neoplasia. 2015 Jan;17(1):1-15.

32 Pauli C, Hopkins BD, Prandi D, Shaw R, Fedrizzi T, Sboner A, et al. Personalized In Vitro and In Vivo Cancer Models to Guide Precision Medicine. Cancer Discov. 2017 May; 7(5):462-77.

33 Tiriac H, Belleau P, Engle DD, Plenker D, Deschênes A, Somerville TD, et al. Organoid Profiling Identifies Common Responders to Chemotherapy in Pancreatic Cancer. Cancer Discov. 2018 Sep;8(9):1112-29.

34 van de Wetering M, Francies HE, Francis JM, Bounova G, Iorio F, Pronk A, et al. Prospective derivation of a living organoid biobank of colorectal cancer patients. Cell. 2015 May; 161(4):933-45.
35 Bankhead P, Loughrey MB, Fernández JA, Dombrowski Y, McArt DG, Dunne PD, et al. QuPath: open source software for digital pathology image analysis. Sci Rep. 2017 Dec; $7(1): 16878$.

36 Sebaugh JL. Guidelines for accurate EC50/ IC50 estimation. Pharm Stat. 2011 Mar-Apr; 10(2):128-34.

37 Garnett MJ, Edelman EJ, Heidorn SJ, Greenman CD, Dastur A, Lau KW, et al. Systematic identification of genomic markers of drug sensitivity in cancer cells. Nature. 2012 Mar; 483(7391):570-5.

38 Wilkerson MD, Hayes DN. ConsensusClusterPlus: a class discovery tool with confidence assessments and item tracking. Bioinformatics. 2010 Jun;26(12):1572-3.

39 UniProt Consortium. UniProt: a worldwide hub of protein knowledge. Nucleic Acids Res. 2019 Jan;47 D1:D506-15.

40 Hilfenhaus G, Göhrig A, Pape UF, Neumann $\mathrm{T}$, Jann $\mathrm{H}$, Zdunek D, et al. Placental growth factor supports neuroendocrine tumor growth and predicts disease prognosis in patients. Endocr Relat Cancer. 2013 May;20(3):305-19.

41 Hughes CS, Postovit LM, Lajoie GA. Matrigel: a complex protein mixture required for optimal growth of cell culture. Proteomics. 2010 May;10(9):1886-90.

42 Kleinman HK, Martin GR. Matrigel: basement membrane matrix with biological activity. Semin Cancer Biol. 2005 Oct;15(5):378-86.

43 Kutschka I, Chen IY, Kofidis T, Arai T, von Degenfeld G, Sheikh Ahmad Y., et al. Collagen matrices enhance survival of transplanted cardiomyoblasts and contribute to functional improvement of ischemic rat hearts. Circulation. 2006 Jul;114(1 suppl):I-167.

44 Poincloux R, Collin O, Lizárraga F, Romao M, Debray M, Piel M, et al. Contractility of the cell rear drives invasion of breast tumor cells in 3D Matrigel. Proc Natl Acad Sci USA. 2011 Feb;108(5): 1943-8

45 Zheng W, Zhang W, Jiang X. Biomimetic collagen nanofibrous materials for bone tissue engineering. Adv Eng Mater. 2010 Sep; 12(9):B451-66.

46 Montesano R, Mouron P, Amherdt M, Orci L. Collagen matrix promotes reorganization of pancreatic endocrine cell monolayers into isletlike organoids. J Cell Biol. 1983 Sep;97(3):935-9.

47 Kaderli RM, Spanjol M, Kollár A, Bütikofer L, Gloy V, Dumont RA, et al. Therapeutic Options for Neuroendocrine Tumors: A Systematic Review and Network Meta-analysis. JAMA Oncol. 2019 Apr;5(4):480-9.

48 Bolck HA, Pauli C, Göbel E, Mühlbauer K, Dettwiler S, Moch H, et al. Cancer sample biobanking at the next level: combining tissue with living cell repositories to promote precision medicine. Front Cell Dev Biol. 2019 Oct; 7:246.

49 Alkema NG, Tomar T, Duiker EW, Jan Meersma G, Klip H, van der Zee AG, et al. Biobanking of patient and patient-derived xenograft ovarian tumour tissue: efficient preservation with low and high fetal calf serum based methods. Sci Rep. 2015 Oct;5(1):14495. 
50 Sato T, Vries RG, Snippert HJ, van de Wetering M, Barker N, Stange DE, et al. Single Lgr5 stem cells build crypt-villus structures in vitro without a mesenchymal niche. Nature. 2009 May;459(7244):262-5.

51 Sato T, Stange DE, Ferrante M, Vries RG, Van Es JH, Van den Brink S, et al. Long-term expansion of epithelial organoids from human colon, adenoma, adenocarcinoma, and Barrett's epithelium. Gastroenterology. 2011 Nov;141(5):1762-72.

52 Fujii M, Shimokawa M, Date S, Takano A, Matano M, Nanki K, et al. A Colorectal Tumor Organoid Library Demonstrates Progressive Loss of Niche Factor Requirements during Tumorigenesis. Cell Stem Cell. 2016 Jun;18(6):827-38.

53 Matano M, Date S, Shimokawa M, Takano A, Fujii M, Ohta Y, et al. Modeling colorectal cancer using CRISPR-Cas9-mediated engineering of human intestinal organoids. Nat Med. 2015 Mar;21(3):256-62.
54 Drost J, van Boxtel R, Blokzijl F, Mizutani T, Sasaki N, Sasselli V, et al. Use of CRISPRmodified human stem cell organoids to study the origin of mutational signatures in cancer. Science. 2017 Oct;358(6360):234-8.

55 Weeber F, Ooft SN, Dijkstra KK, Voest EE. Tumor Organoids as a Pre-clinical Cancer Model for Drug Discovery. Cell Chem Biol. 2017 Sep;24(9):1092-100.

56 Kojima N. In vitro reconstitution of pancreatic islets. Organogenesis. 2014 Apr-Jun; 10(2):225-30.

57 Koumarianou A, Kaltsas G, Kulke MH, Oberg K, Strosberg JR, Spada F, et al. Temozolomide in Advanced Neuroendocrine Neoplasms: Pharmacological and Clinical Aspects. Neuroendocrinology. 2015;101(4):274-88.

58 Fu D, Calvo JA, Samson LD. Balancing repair and tolerance of DNA damage caused by alkylating agents. Nat Rev Cancer. 2012 Jan; 12(2):104-20.

59 Lohse I, Al-Ali H, Volmar CH, D Alvarez Trotta A, Brothers SP, Capobianco AJ, et al. Ex vivo drug sensitivity testing as a means for drug repurposing in esophageal adenocarcinoma. PLoS One. 2018 Sep;13(9):e0203173.
60 Majumder B, Baraneedharan U, Thiyagarajan S, Radhakrishnan P, Narasimhan H, Dhandapani $\mathrm{M}$, et al. Predicting clinical response to anticancer drugs using an ex vivo platform that captures tumour heterogeneity. Nat Commun. 2015 Feb;6(1):6169.

61 Naipal KA, Verkaik NS, Ameziane N, van Deurzen CH, Ter Brugge P, Meijers M, et al. Functional ex vivo assay to select homologous recombination-deficient breast tumors for PARP inhibitor treatment. Clin Cancer Res. 2014 Sep;20(18):4816-26.

62 Garralda E, Paz K, López-Casas PP, Jones S, Katz A, Kann LM, et al. Integrated next generation sequencing and avatar mouse models for personalized cancer treatment. Clin Cancer Res. 2014 May;20(9):2476-84.

63 Weroha SJ, Becker MA, Enderica-Gonzalez S, Harrington SC, Oberg AL, Maurer MJ, et al. Tumor grafts as in vivo surrogates for women with ovarian cancer. Clin Cancer Res. 2014 Mar;20(5):1288-97. 